\title{
Constructing an index of physical fitness age for Japanese elderly based on 7-year longitudinal data: sex differences in estimated physical fitness age
}

\author{
Misaka Kimura • Chinatsu Mizuta • \\ Yosuke Yamada Y Yasuko Okayama • \\ Eitaro Nakamura
}

Received: 1 October 2010 / Accepted: 21 February 2011/Published online: 22 March 2011

(C) The Author(s) 2011. This article is published with open access at Springerlink.com

\begin{abstract}
A standardized method for assessing the physical fitness of elderly adults has not yet been established. In this study, we developed an index of physical fitness age (fitness age score, FAS) for older Japanese adults and investigated sex differences based on the estimated FAS. Healthy elderly adults ( 52 men, 70 women) who underwent physical fitness tests once yearly for 7 years between 2002 and 2008 were included in this study. The age of the participants at the beginning of this study ranged from 60.0 to 83.0 years. The physical fitness tests consisted of 13 items to measure balance, agility, flexibility, muscle strength, and endurance. Three criteria were used to evaluate fitness markers of aging: (1) significant
\end{abstract}

Electronic supplementary material The online version of this article (doi:10.1007/s11357-011-9225-5) contains supplementary material, which is available to authorized users.

M. Kimura $(\bowtie) \cdot$ C. Mizuta $\cdot$ Y. Yamada $\cdot$ Y. Okayama

Kyoto Prefectural University of Medicine,

Kyoto, Japan

e-mail: misaka@cmt.kpu-m.ac.jp

Y. Yamada

The Fukuoka University Institute for Physical Activity and Global Fukuoka University Program,

Fukuoka, Japan

E. Nakamura $(\triangle)$

Department of Sport Science,

Kyoto Iken College of Medicine and Health,

Kyoto, Japan

email: naka-e@kyoto-iken.ac.jp cross-sectional correlation with age; (2) significant longitudinal change with age consistent with the cross-sectional correlation; and (3) significant stability of individual differences. We developed an equation to assess individual FAS values using the first principal component derived from principal component analysis. Five candidate fitness markers of aging (10-m walking time, functional reach, one leg stand with eyes open, vertical jump and grip strength) were selected from the 13 physical fitness tests. Individual FAS was predicted from these five fitness markers using a principal component model. Individual FAS showed high longitudinal stability for age-related changes. This investigation of the longitudinal changes of individual FAS revealed that women had relatively lower physical fitness compared with men, but their rate of physical fitness aging was slower than that of men.

Keywords Physical fitness age - Sex differences . Biomarker of aging $\cdot$ Mobility disability $\cdot$ Frailty $\cdot$ Gait speed

\section{Introduction}

Previous studies have shown that physical fitness is a strong independent predictor of mortality (Fujita et al. 1995; Newman et al. 2006; Takata et al. 2007; Park et al. 2009). Because individuals with high physical fitness may have greater muscle mass and quality (Lauretani et al. 2003; Yamada et al. 2010), and 
greater reserve capacity to resist physical stress (Astrand 1956; Piscopo 1985), improvements in the physical fitness of elderly individuals through appropriate physical activity should enable such individuals to maintain their daily activity into later years of life.

Since Furukawa et al. (1975) described a method in 1974 to estimate fitness age from physical functions using multiple regression analysis, various other estimation methods have been reported (Heikkinen and Kayhty 1977; Suominen 1978; Hofecker et al. 1980; Nakamura et al. 1989, 1990, 1996), which also use physical fitness age estimated from health-related physical fitness test scores using cross-sectional data. Such approaches based on physical fitness age are advantageous and useful because they allow us to intuitively evaluate the individual's corresponding physical fitness age (Nakamura et al. 1989). However, a standardized method for assessing physical fitness age using longitudinal data has not yet been established.

Therefore, the purpose of this study was to identify candidate fitness markers of aging and construct an index of physical fitness age (FAS, fitness age score) in elderly Japanese individuals using 7-year longitudinal data, and to investigate sex differences in physical fitness based on the estimated FAS.

\section{Methods}

Participants

Among 2,844 elderly Japanese individuals who underwent routine physical fitness tests between
2002 and 2008 at the gymnasium of Kyoto Prefecture University of Medicine (Yamada et al. 2010), 122 healthy elderly adults (52 men and 70 women) aged 60 years and over underwent a 1-day routine physical fitness test every year for 7 years between 2002 and 2008 and were randomly selected as subjects. Characteristics, including past and present health status, physical activity status, and social and dietary habits, were collected from a medical questionnaire. All participants read and signed an institutionally approved informed consent form before participating in this study.

Most participants resided in Kyoto City. Their occupations included managers $(3.0 \%)$, researchers $(2.0 \%)$, teachers $(2.0 \%)$, storekeepers $(4.0 \%)$, and unemployed $(89.0 \%)$, in men, while most women were unemployed. The age of the participants at the beginning of this study (2002) ranged from 60.0 to 83.0 years, with a mean age of 73.8 years for men and 72.3 years for women. The characteristics of the participants divided into three age groups (60-69, 7079 , and $\geq 80$ years old) are given in Table 1 .

Test items

A battery of physical function tests designed for adults aged $\geq 60$ years old (Kimura et al. 1989, 1990, 1994, 1998; Kaneko et al. 1991; Kimura and Adachi 1999; Yamada et al. 2010) was used to measure physical fitness. The battery of tests included 13 items to measure balance, agility, flexibility, muscle strength, and endurance as described in Table 2. These test items were devised to be suitable and safe
Table 1 Age and physical characteristics of the participants at baseline

\begin{tabular}{lccr}
\hline \multicolumn{3}{c}{ Age group (year) } & \\
\cline { 2 - 4 } & $60-69$ & $70-79$ & $\geq 80+$ \\
\hline Men & & & \\
$n$ & 10 & 34 & $82.1 \pm 2.40$ \\
Age (year), mean \pm SD & $66.3 \pm 2.28$ & $74.3 \pm 2.84$ & $159.0 \pm 7.43$ \\
Height (m), mean \pm SD & $166.1 \pm 5.09$ & $163.1 \pm 5.58$ & $56.1 \pm 9.45$ \\
Weight (kg), mean \pm SD & $61.5 \pm 4.88$ & $60.1 \pm 8.45$ & 11 \\
Women & & & $82.0 \pm 2.00$ \\
$n$ & 25 & 34 & $148.7 \pm 4.28$ \\
Age (year), mean \pm SD & $66.1 \pm 2.29$ & $74.2 \pm 2.85$ & $47.4 \pm 6.06$ \\
Height (m), mean \pm SD & $152.6 \pm 4.43$ & $149.6 \pm 4.93$ & $50.3 \pm 7.03$ \\
Weight (kg), mean \pm SD & $52.2 \pm 7.03$ & & \\
\hline
\end{tabular}


Table 2 Correlation coefficients obtained from cross-sectional, longitudinal, and stability analyses of healthy older men and women

\begin{tabular}{|c|c|c|c|c|c|c|}
\hline \multirow[t]{2}{*}{ Variable } & \multicolumn{2}{|c|}{ Cross-sectional analysis } & \multicolumn{2}{|c|}{ Longitudinal analysis } & \multicolumn{2}{|c|}{ Stability analysis } \\
\hline & Men $(n=52)$ & Women $(n=70)$ & Men $(n=52)$ & Women $(n=70)$ & Men $(n=52)$ & Women $(n=70)$ \\
\hline 1. One leg stand with eyes open & $-0.294 *$ & $-0.377 * *$ & $-0.275^{*}$ & $-0.297 * *$ & $0.629 * *$ & $0.711^{* *}$ \\
\hline 2. One leg stand with eyes closed & -0.154 & -0.208 & -0.179 & -0.203 & $0.479 * *$ & $0.631 * *$ \\
\hline 3. Functional reach test & $-0.322 *$ & $-0.330 * *$ & $-0.286^{*}$ & $-0.227 *$ & $0.486 * *$ & $0.417 * *$ \\
\hline 4. Chair stepping & -0.160 & $-0.222 *$ & -0.102 & 0.058 & $0.780 * *$ & $0.593 * *$ \\
\hline 5. Trunk flexion & -0.151 & -0.212 & -0.150 & -0.180 & $0.796 * *$ & $0.718 * *$ \\
\hline 6. Leg strength & $-0.416^{* *}$ & $-0.332 * *$ & $-0.271^{*}$ & -0.064 & $0.857^{* *}$ & $0.664 * *$ \\
\hline 7. Grip strength & $-0.352 * *$ & $-0.370 * *$ & $-0.546^{* *}$ & $-0.398 * *$ & $0.861^{* *}$ & $0.847 * *$ \\
\hline 8. Vertical jump & $-0.529 * *$ & $-0.580 * *$ & $-0.456^{* *}$ & $-0.514 * *$ & $0.729 * *$ & $0.768^{* *}$ \\
\hline 9. Shuttle stamina walk test & $-0.352 * *$ & $-0.460 * *$ & -0.205 & -0.190 & $0.819 * *$ & $0.762 * *$ \\
\hline 10. 6-m walk (speed) & -0.223 & $-0.411 * *$ & $-0.303 *$ & -0.108 & $0.690 * *$ & $0.776^{* *}$ \\
\hline 11. 6-m walk (footsteps) & $-0.309 *$ & $-0.391 * *$ & $-0.291 *$ & -0.181 & $0.406^{* *}$ & $0.511^{* *}$ \\
\hline 12. $10-\mathrm{m}$ walk time & $0.323^{*}$ & $0.389 * *$ & $0.538^{* *}$ & $0.383^{* *}$ & $0.554 * *$ & $0.553^{* *}$ \\
\hline 13. Chair stand & 0.234 & $0.354 * *$ & 0.124 & $0.340 * *$ & $0.791 * *$ & $0.745^{* *}$ \\
\hline
\end{tabular}

$* P<0.05 ; * * P<0.01$

for elderly individuals who had not exercised for a long time. The supplementary material provides further detail about the tests performed. All of the test items have been validated for use in older adults, and each item has demonstrated acceptable test-retest reliability, with correlation coefficients ranging from 0.70 to 0.90 (Kimura and Adachi 1999).

\section{Statistical analysis}

All analyses, including systematic and logical selection of candidate fitness markers of aging, principle component analysis, and $t$ tests for comparison of slopes, were done using Statistical Package for the Social Sciences software (SPSS Inc., Chicago, IL, USA).

\section{Definition of FAS}

The FAS represents the physical fitness status of a normal person at a certain chronological age (CA). Whether a person's fitness age is lower or higher than his CA indicates the ability of the individual to function efficiently and safely in everyday activities of work and play without experiencing fatigue and with sufficient energy reserves to cope with unexpected physical demands (Piscopo 1985).

\section{Estimation of the FAS}

In this study, we developed an equation for FAS using the first principal component obtained from principal component analysis. In general, the first principal component, which accounts for the largest variance in the extracted components, is a useful statistical tool to combine all of the explanatory variables into a single expression in the absence of a dependent variable (Harman 1967). Our method for predicting biological or physical fitness age has been described in more detail elsewhere (Nakamura 1994).

\section{Results}

Selection of candidate fitness markers for aging

For systematic and logical selection of candidate fitness markers of aging, we used the following stepwise methods: (1) cross-sectional analysis, (2) longitudinal analysis, and (3) stability analysis. This method was developed by Ingram et al. (2001) and has been used to identify biomarkers of aging in humans.

Table 2 summarizes the correlations between factors used to guide the first three steps of the 
selection process applied to identity the candidate fitness markers of aging.

Step 1: Cross-sectional analysis.

To identify the magnitude of the association between each variable and CA, we first examined cross-sectional data for each year (2002-2008). The values of 13 physical fitness tests were correlated with CA for each participant for each year across all age groups. Thus, we produced seven correlations for each variable. The Pearson product moments for each collection obtained for each year were then averaged to obtain an estimate of the mean cross-sectional correlation with CA using Fisher's $\mathrm{r}$ to $z$ and $z$ to $r$ transformations. Based on this criterion, we identified eight variables for further analysis for men: one-leg stand with eyes open, vertical jump, grip strength, shuttle stamina walk test (SSTw), functional reach, 6-m walk (number of footsteps), 10-m walk (time), and leg strength $(P<0.05)$. For women, we identified the following 11 variables for further analysis: one leg stand with eyes open, vertical jump, chair stepping, grip strength, SSTw, functional reach, 6-m walk (speed), 6-m walk (number of footsteps), 10-m walk (time), leg strength, and chair stand $(P<0.05)$.

Step 2: Longitudinal analysis

To identify the magnitude of genuine agerelated changes in each variable, we first transformed the measurement variable and CA for each subject across 7 years to $z$ scores to standardize the scales. We then calculated correlations between $\mathrm{CA}$ and the values for each subject. Using Fisher's $r$ to $z$ and $z$ to $r$ transformations, we calculated the means of the individual $r$ values for all subjects. Based on this analysis, we identified eight variables for further analysis in men: one leg stand with eyes open, vertical jump, grip strength, functional reach, 6-m walk (speed), 6-m walk (number of footsteps), 10-m walk (time), and leg strength $(P<0.05)$. For women, we identified six variables for further analysis: one leg stand with eyes open, vertical jump, grip strength, functional reach, 10-m walk (time), and chair stand $(P<0.05)$.

Step 3: Stability analysis

We next examined the longitudinal stability of individual differences for all variables. For this analysis, we evaluated the inter-year reliability of the annual values for each variable. We determined correlations between the measurement value obtained for each variable and the corresponding value for the subsequent year, i.e., between 2002 and 2003, and between 2003 and 2004, for example, across all ages within subjects. To calculate a mean value of the correlation coefficients across the 7 years, we applied Fisher's $r$ to $z$ and $z$ to $r$ transformations. The measurements for all variables evaluated were stable, ranging from 0.406 for the $6-\mathrm{m}$ walk (number of footsteps) to 0.861 for grip strength in men, and from 0.417 for functional reach to 0.847 for grip strength in women. The stability coefficient for tests with complicated movements tended to show lower coefficients than test with simple movements.

Comparing the results of steps 1 and 2, the seven variables that met the criteria for significant longitudinal changes with age consistent with their crosssectional correlations for men were one leg stand with eyes open, vertical jump, grip strength, functional reach, 6-m walk (number of footsteps), 10-m walk (time), and leg strength. For women, we identified six variables: one leg stand with eyes open, vertical jump, grip strength, functional reach, 10-m walk (time) and chair stand. These variables were carefully selected to select a candidate variable included in the models for both men and women. In the comparison of models for men and women, three items (6-m walk number of footsteps) and leg strength for men, and chair stand for women) did not show an age-related change and were removed.

Based on these statistical analyses, five variablesone leg stand with eyes open, vertical jump, grip strength, functional reach, 10-m walk (time)-were assessed as candidate fitness markers for aging. The data obtained for the five candidate fitness markers of aging across the 7 years of the study are presented in Fig. 1 (men) and Fig. 2 (women) as functions of CA. 

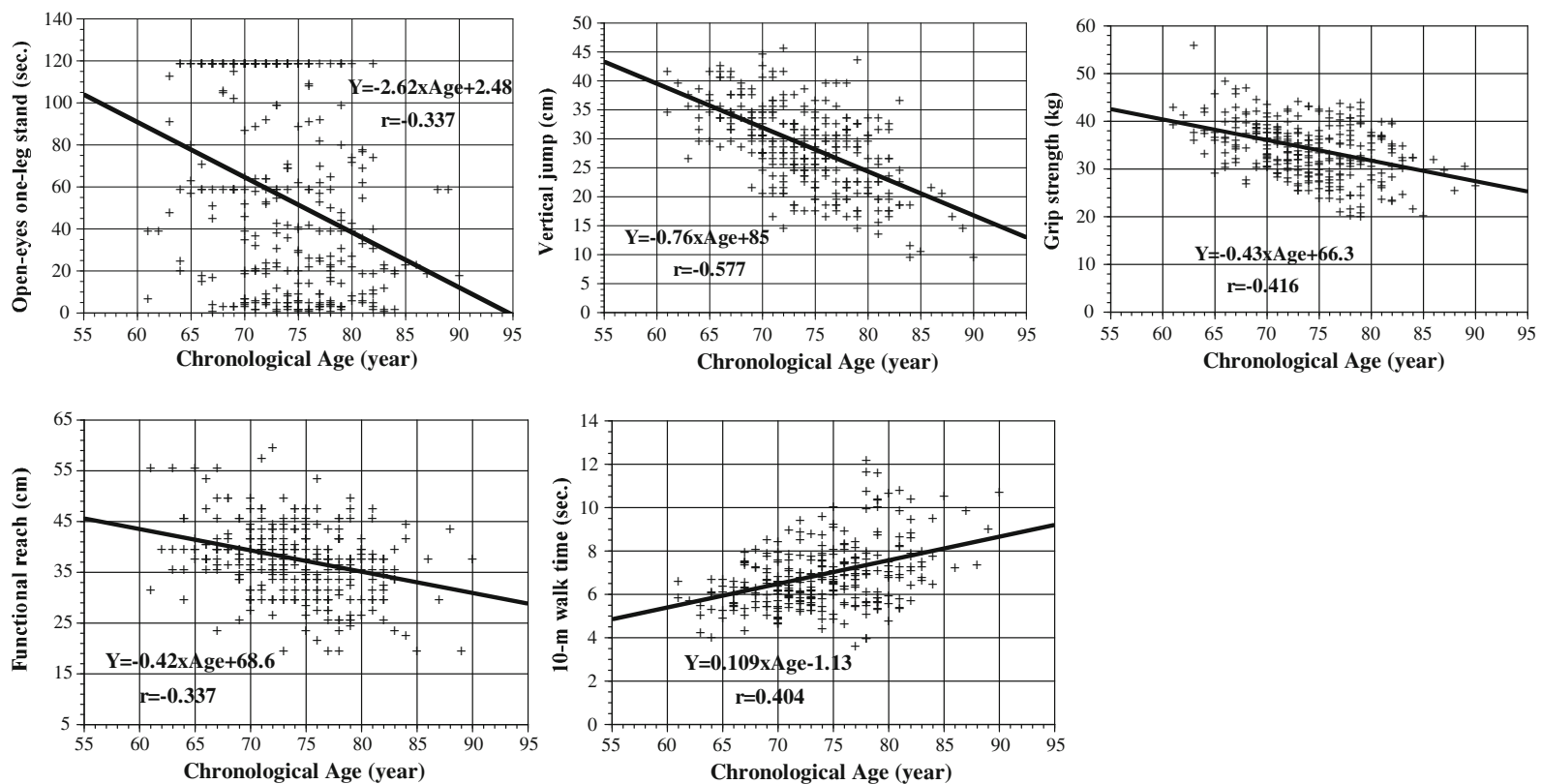

Fig. 1 Scatter plots and regression lines of the five candidate fitness markers of aging in men based on cross-sectional data analysis: one leg stand with eyes open, vertical jump, grip strength, functional reach, and 10-m walk time. The figure shows the results obtained from 7-year longitudinal data for 52 older men

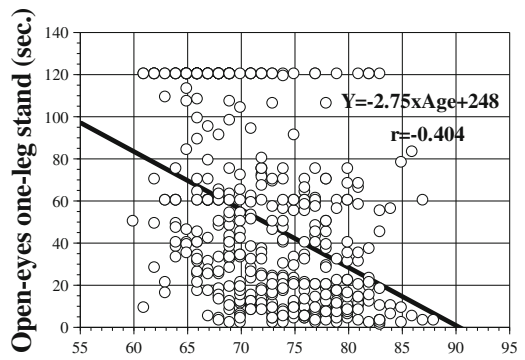

Chronological Age (year)

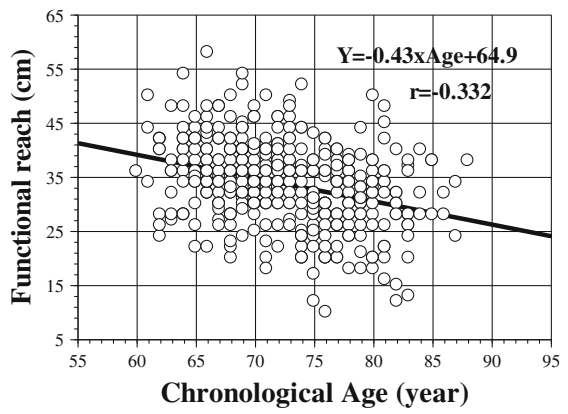

Fig. 2 Scatter plots and regression lines of the five candidate fitness markers of aging in women: one leg stand with eyes open, vertical jump, grip strength, functional reach, and 10-m
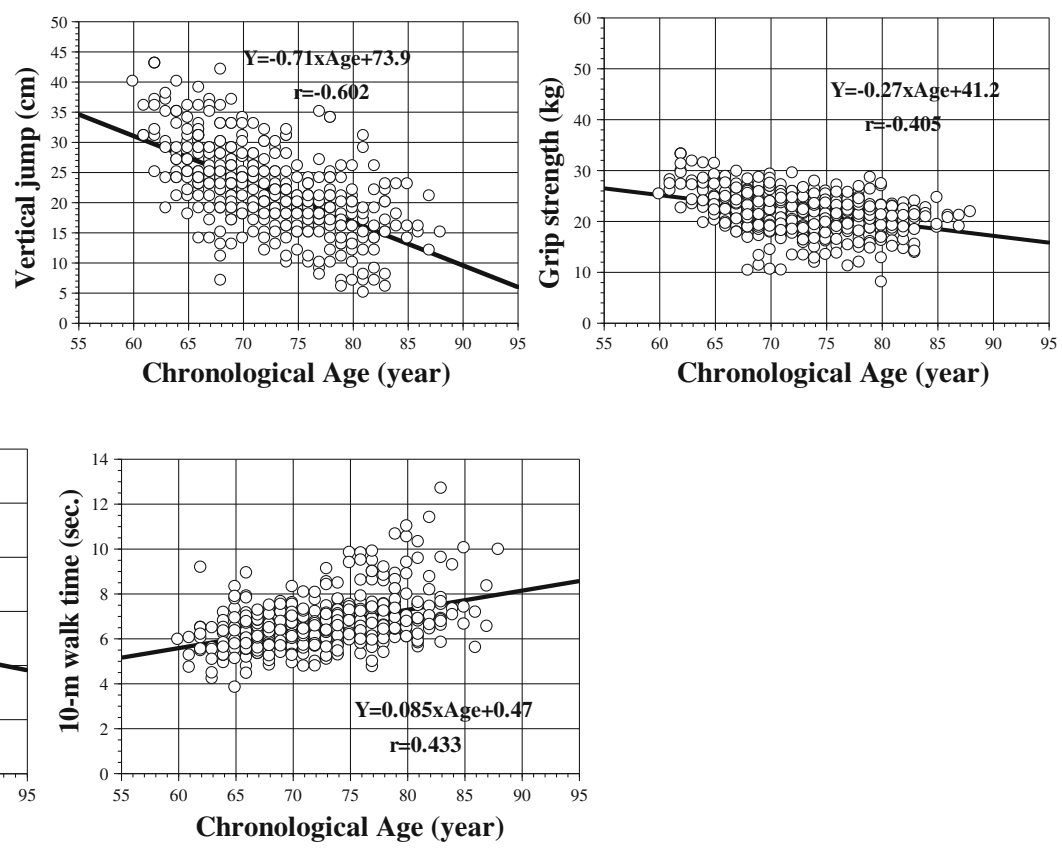

walk time. The figure shows the results obtained from 7-year longitudinal data for 70 older women 


\section{Estimation of the FAS}

Principal component analysis was applied to the correlation matrix of the five candidate fitness markers of aging identified above (Tables 3 and Table 4). This analysis was conducted to determine the structure of covariance. For the first analysis, CA was included to confirm the relationship between age and the principal component identified. For the second analysis, CA was excluded to ascertain whether the relationships of the candidate fitness markers of aging to the principal components remained without an influence of CA.

The first analysis identified one major principal component in both sexes with corresponding Eigenvalues greater than 1.0 based on recommended criteria (Guttman 1954). The results of these analyses are shown in Table 5 . About $50 \%$ of the total variance was accounted for by one principal component. CA shows an extremely high factor loading (0.743) with this component. Meanwhile, all of the candidate fitness markers for aging showed significant loading on the first principal component, explaining $49.5 \%$ of the total variance for men and $46.5 \%$ for women. Therefore, the first principal component appeared to be a major age factor.

Results from the second analysis presented in Table 6 revealed that all of the candidate fitness markers maintained their significant factor loadings on the first principal component, even when CA was removed from the model. Moreover, this component maintained a high level of the total variance $(49.4 \%$ for men and $44.6 \%$ for women). From these results, we deduced that the five candidate fitness-markersone leg stand with eyes open, vertical jump, grip strength, functional reach and $10-\mathrm{m}$ walk (time) represented an underlying factor that might reflect the aging process of physical fitness.
Constructing the FAS

Because the five candidate fitness markers of aging were considered to measure the underlying aging processes for physical fitness, we proceeded to combine them into a multivariate index, designated the FAS. To calculate individual FAS values, each test score for an individual was first standardized and then summed across tests in a weighted manner using the coefficients of the factor scores obtained in the principle component analysis. In this procedure, we reduced the equation used to calculate individual FAS value to a simple equation, as follows:

Men:

$$
\begin{aligned}
\mathrm{FAS}= & -0.203 X_{1}+0.034 X_{2}+0.0064 X_{3}+0.044 X_{4} \\
& +0.046 X_{5}-3.05
\end{aligned}
$$

Women:

$$
\begin{aligned}
\text { FAS }= & -0.263 X_{1}+0.033 X_{2}+0.0074 X_{3}+0.048 X_{4} \\
& +0.079 X_{5}-2.52
\end{aligned}
$$

where, $X_{1}=10$-m walk (time) (s), $X_{2}=$ functional reach (cm), $X_{3}=$ one leg stand with eyes open (s), $X_{4}=$ vertical jump (cm), and $X_{5}=$ grip strength $(\mathrm{kg})$.

The individual FAS values for 52 men based on the 7-year longitudinal data were calculated using the equation for men. Figure 3 shows the scatter plot for FAS value with CA for all male subjects. In the cross-sectional analysis based on the 7-year longitudinal data (287 values), the individual FAS values were scattered relatively symmetrically above and below the regression line. The correlation coefficient between the FAS values and CA was $0.59(P<0.01)$, and the standard error of the estimate (SEE) was 0.81 .
Table 3 Means, standard deviations (SD) and correlation matrices of five markers of aging and chronological age calculated from 7-year longitudinal data for 52 healthy older men

\begin{tabular}{lrrrrrrrr}
\hline Variable & Means & SD & \multicolumn{1}{l}{1} & 2 & 3 & 4 & 5 & 6 \\
\hline 1. Age (year) & 73.9 & 5.4 & & & & & \\
2. 10-m walk time (s) & 6.9 & 1.46 & 0.404 & & & & \\
3. Functional reach test (cm) & 37.4 & 7.18 & -0.337 & -0.260 & & & \\
4. One leg stand with eyes open (s) & 53.5 & 44.2 & -0.317 & -0.377 & 0.306 & & \\
5. Vertical jump (cm) & 28.9 & 7.2 & -0.577 & -0.464 & 0.348 & 0.490 & \\
6. Grip strength (kg) & 34.4 & 5.9 & -0.416 & -0.453 & 0.325 & 0.267 & 0.363 \\
\hline
\end{tabular}


Table 4 Means, standard deviations (SD) and correlation matrices of five markers of aging and chronological age calculated from 7-year longitudinal data for 70 healthy older women

\begin{tabular}{lrrrrrrrr}
\hline Variable & Means & SD & \multicolumn{1}{l}{1} & 2 & 3 & 4 & 5 & 6 \\
\hline 1. Age (year) & 72.5 & 5.8 & & & & & \\
2. 10-m walk time (s) & 6.7 & 1.23 & 0.433 & & & & \\
3. Functional reach test (cm) & 33.6 & 7.73 & -0.332 & -0.236 & & & \\
4. One leg stand with eyes open (s) & 49.5 & 40.1 & -0.404 & -0.250 & 0.241 & & \\
5. Vertical jump (cm) & 22.1 & 7.0 & -0.602 & -0.376 & 0.311 & 0.369 & \\
6. Grip strength (kg) & 21.9 & 3.9 & -0.405 & -0.340 & 0.206 & 0.337 & 0.371 \\
\hline
\end{tabular}

Similarly, we calculated the individual FAS values for 70 women based on the 7-year longitudinal data using the equation for women (416 values). Figure 4 shows the scatter plot for FAS values with CA for all women. As in men, the individual FAS values were scattered relatively symmetrically above and below the regression line. The correlation coefficient between FAS values and CA was $0.67(P<0.01)$ and the SEE was 0.74.

\section{Sex differences in FAS}

We next investigated the sex differences in the FAS values. To achieve this, we recalculated the FAS values for the 70 women using the equation for men described above and they were overlaid on the scatter plot for men in Fig. 3. As shown in Fig. 5, the FAS values for women are scattered below the regression line of the FAS values for men, suggesting that women are less physically fit than men at the same age. The differences between men and women in estimated FAS values may depend on differences in physique. To examine the influence of the physique of women on these differences, we calculated the difference in FAS values of women from the regression line for men. Next, a multiple regression analysis was applied to the correlation matrix calcu- lated from two variables (height and body mass) and the deviations. The coefficient for multiple correlations of these two variables with the deviations was $0.353[F=27.2$; degrees of freedom $(d f)=415$ and 2 ; $P<0.001]$. However, the ratio of this coefficient to the total variance was only $0.12(12 \%)$, suggesting that physique does not strongly influence the FAS values. However, Fig. 5 does not reflect the individual state of age-related physical fitness because of the crosssectional data analyses.

To examine the changes in aging of older adults based on the estimated FAS values, regression lines for the FAS values on CA for all 52 men and 70 women across all 7 years were calculated and are presented in Figs. 6 and 7. In these figures, the individual estimates of FAS were removed to avoid complex graphical representation; only the regression lines are plotted. The change in the aging ratio for FAS tends to show an exponential curve rather than a straight line. Thus, the subjects were divided into three age groups (60-69, $70-79$, and $\geq 80$ years) based on the participants' ages at 3.5 years (halfway through the study). The mean slopes of the regression lines of FAS values on CA for the three age groups were $-0.163,-0.190$, and -0.367 for men, and $-0.196,-0.162$, and -0.221 for women, respectively. For men, one-way analysis of variance of
Table 5 Principal component analysis of five candidate markers for physical fitness and chronological age

\footnotetext{
${ }^{a}$ First principal component

${ }^{\mathrm{b}}$ First principal component coefficient
}

\begin{tabular}{|c|c|c|c|c|}
\hline \multirow[t]{2}{*}{ Variable } & \multicolumn{2}{|l|}{ Men } & \multicolumn{2}{|l|}{ Women } \\
\hline & $\mathrm{P} 1^{\mathrm{a}}$ & $\mathrm{F} 1^{\mathrm{b}}$ & $\mathrm{P} 1$ & $\mathrm{~F} 1$ \\
\hline Age (y) & -0.743 & -0.255 & -0.819 & -0.293 \\
\hline 10-m walk time (s) & -0.712 & -0.244 & -0.643 & -0.230 \\
\hline Functional reach test $(\mathrm{cm})$ & 0.590 & 0.202 & 0.531 & 0.190 \\
\hline One leg stand with eyes open (s) & 0.654 & 0.224 & 0.629 & 0.225 \\
\hline Vertical jump (cm) & 0.797 & 0.273 & 0.769 & 0.275 \\
\hline Grip strength (kg) & 0.671 & 0.230 & 0.664 & 0.238 \\
\hline Eigenvalue & 2.918 & & 2.793 & \\
\hline$\%$ total variance & 49.46 & & 46.54 & \\
\hline
\end{tabular}


Table 6 Principal component analysis of five candidate markers for physical fitness

${ }^{\text {a }}$ First principal component

${ }^{\mathrm{b}}$ First principal component coefficient

\begin{tabular}{|c|c|c|c|c|}
\hline \multirow[t]{2}{*}{ Variable } & \multicolumn{2}{|l|}{ Men } & \multicolumn{2}{|l|}{ Women } \\
\hline & $\mathrm{P} 1^{\mathrm{a}}$ & $\mathrm{F} 1^{\mathrm{b}}$ & $\mathrm{P} 1$ & $\mathrm{~F} 1$ \\
\hline 10-m walk time (s) & -0.740 & -0.299 & -0.663 & -0.298 \\
\hline Functional reach test $(\mathrm{cm})$ & 0.610 & 0.247 & 0.563 & 0.253 \\
\hline One leg stand with eyes open (s) & 0.698 & 0.282 & 0.660 & 0.296 \\
\hline Vertical jump (cm) & 0.777 & 0.314 & 0.752 & 0.337 \\
\hline Grip strength (kg) & 0.680 & 0.275 & 0.687 & 0.308 \\
\hline Eigenvalue & 2.473 & & 2.228 & \\
\hline$\%$ total variance & 49.46 & & 44.56 & \\
\hline
\end{tabular}

the mean slopes for the three age groups confirmed a significant group effect $(F=7.374 ; d f=49$ and 2; $P<0.01)$. Furthermore, analysis of the differences between the pairs of means using Tukey's method revealed significant differences $(P<0.05)$ for $60-69$ vs. $\geq 80$ age groups, and for $70-79$ vs. $\geq 80$ age groups. By contrast, for women, we found no statistically significant differences in the mean slopes among the three age groups $(F=1.638 ; d f=67$ and $2 ; P=\mathrm{NS})$. These results suggest that the aging rate of the physical fitness is faster in men than in women. In particular, after 80 years old, the rate of aging rapidly advance $(-0.367$ for men and -0.221 for women).

\section{Discussion}

A logical strategy for identifying candidate biomarkers of aging and to evaluate their reliability and validity has been proposed for rhesus monkeys (Nakamura et al. 1994, 1998; Ingram et al. 2001). In those studies, four criteria

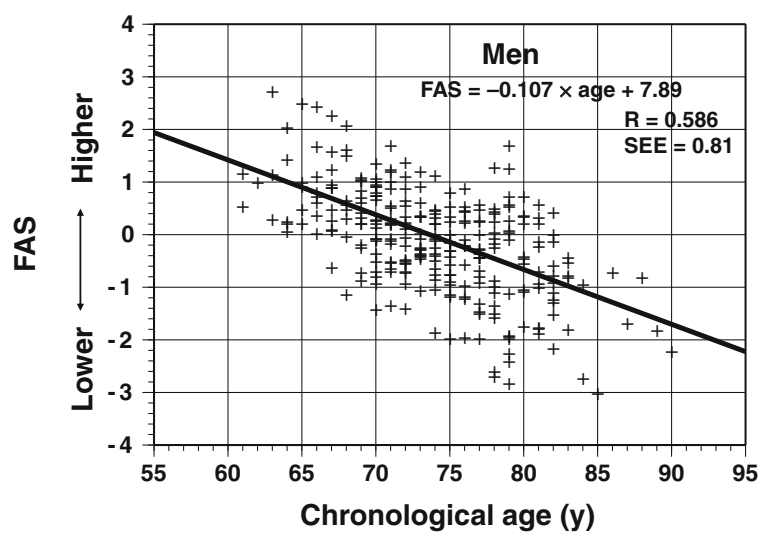

Fig. 3 Relationship between fitness age score (FAS) and chronological age in a cross-sectional analysis of 52 older men were used to evaluate the biomarkers of aging: (1) significant cross-sectional correlation with age, (2) significant longitudinal change with age consistent with the cross-sectional correlation, (3) significant stability of individual differences, and (4) the rate of the age-related change was proportional to differences in lifespan among related species. In this study, we applied the first three criteria to human data to identify candidate fitness markers of aging and identified five fitness markers in men and women: one leg stand with eyes open, vertical jump, grip strength, functional reach, and 10-m walk (time). These five fitness markers represent elements of physical fitness including static balance, leg power, arm strength, dynamic balance, and gross body coordination agility, respectively. In older adults, it has been reported that hip fractures caused by falls have increased (Binder et al. 2004; Fujita et al. 2006). Therefore, balance and leg muscle strength are needed to prevent falls and were assessed in a battery of tests of physical fitness in this study. Thus, evaluating physical fitness in this way would not only aid health

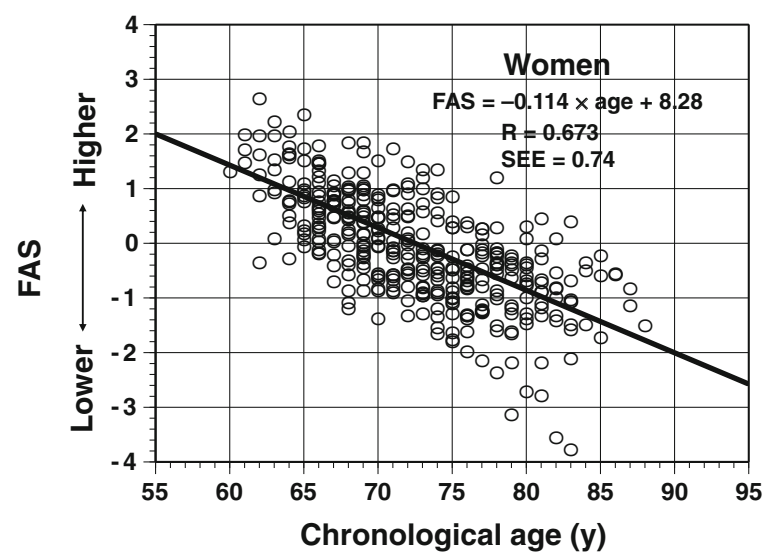

Fig. 4 Relationship between fitness age score (FAS) and chronological age in a cross-sectional analysis of 70 older women 


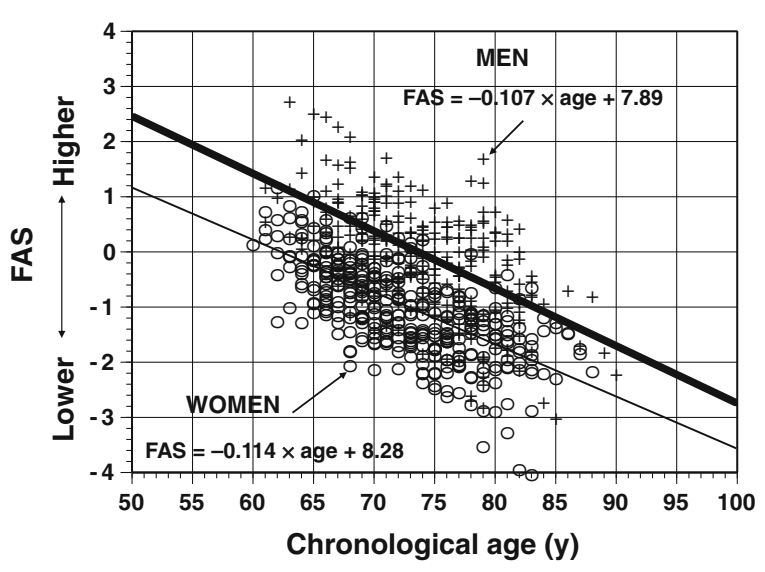

Fig. 5 Comparison of the fitness age scores (FAS) between 70 women and 52 men in a cross-sectional analysis

maintenance, but also reduce the risk of falls in older adults. Furthermore, these fitness tests showed age-related changes in physical fitness. Accordingly, these fitness markers are considered to represent the vital elements of health-related physical fitness.

There is notable overlap between the constructed FAS in the present study and the operational definitions of frailty index (Fried et al. 2001; Searle et al. 2008), as each includes grip strength and walking speed. The recent findings strongly suggest walking speed and grip strength are strong risk factor in the elderly (Cooper et al. 2010; Studenski et al. 2011). It is very interesting, because we used the data in healthy elderly who volunteered to participate in the battery test over 7 years, and also did not examine any

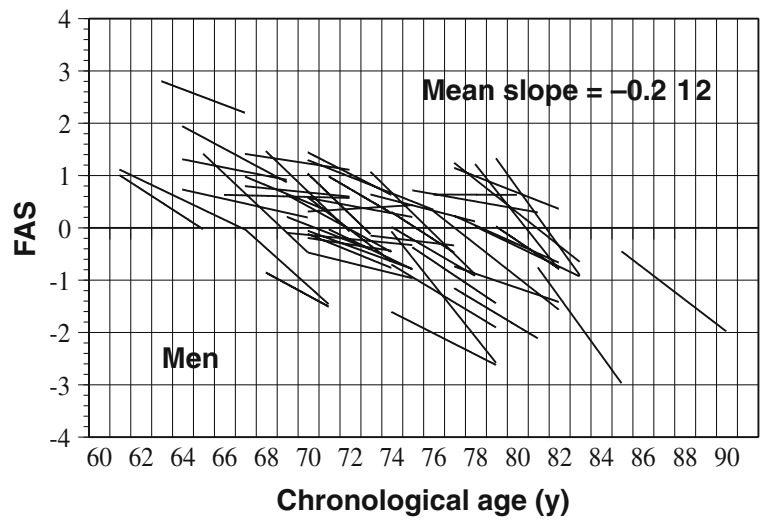

Fig. 6 Association between fitness age score (FAS) and chronological age in a longitudinal analysis of 52 older men. To aid interpretation, only the regression lines for FAS with age are shown in this figure

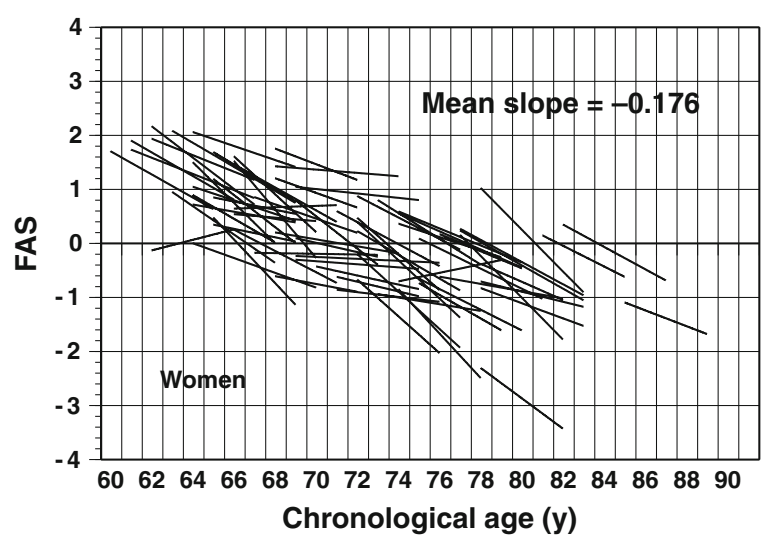

Fig. 7 Association between fitness age score (FAS) and chronological age in a longitudinal analysis of 70 older women. To aid interpretation, only the regression lines for FAS with age are shown in this figure

relationship between the physical fitness age and frailty, functional disability, or mortality. We just examine the significance of cross-sectional correlation, 7-year longitudinal change, and stability of the variables. Our study support the idea that the measurement of hand grip strength and walking speed is useful tool to evaluate healthy aging. In our results, vertical jump is also good to assess fitness age in the elderly.

After confirming the underlying relationship among the five candidate fitness markers, we constructed the FAS index for each individual using weighted loadings of each variable onto the factor score derived from the PCA. We noted that the individual FAS values were scattered relatively symmetrically above and below the regression line for FAS value on CA. The evaluation of physical fitness according to the FAS is advantageous and useful because it allows us to estimate the individuals physical fitness age. However, physical fitness is also affected by phenotypic factors such as exercise, lifestyle habits and environmental circumstances (Piscopo 1985). In 1956, Astrand (1956) proposed that the physical fitness of an individual is determined by his/her natural phenotype and by the way this phenotype has been adapted to training conditions. Thus, an individual's FAS value is not only affected by genetic factors but also by lifestyle factors. Therefore, the difference between the predicted FAS value and actual age reflects the physical fitness level corresponding to the individual's phenotype and the impact of physical activity and training on physical fitness level.

Some researchers have reported that the difference between the physical fitness age and actual age 
reflects the degree of aging (Hollingsworth et al. 1965; Furukawa et al. 1975; Voitenko and Tokar 1983; Dubina et al. 1984; Nakamura et al. 1989). Recently, Nakamura and Miyao (2008) suggested that individual differences are characterized by three factors-age, peak functional capacity, and aging rate-by investigating an index of biological age in humans. Furthermore, they suggested that the peak functional capacity is determined by genetic factors and the aging rate is influenced by environmental factors. When we consider their comments, the difference between the estimated FAS value and actual age does not necessarily reflect an individual's aging rate of physical fitness. Aging can be viewed as a time-dependent complex, integrating primary and secondary aging processes (Hofecker et al. 1980). Thus, based on this 7-year longitudinal data analysis, we investigated the age-related changes in FAS to assess whether the rate of change differed with advancing age. We found that the rate of aging in older men represented by the FAS value was not constant, but rather increased exponentially with advancing age.

To search for possible sex differences in the FAS, we compared the FAS values between the sexes. The FAS values of 70 healthy older women were also calculated using the equation for men established in this study, and overlaid these values on the scatter plot for men $(n=52)$. The FAS values in most of women were distributed below the regression line for FAS values on $\mathrm{CA}$ in men. Furthermore, the mean FAS value for women was much lower than that of men $(0.003 \pm 0.99$ for men vs. $-0.962 \pm 0.829$ for women $)$. These characteristics were maintained even when we considered the influence of physique on physical fitness. Based on these results, the physical fitness level of the woman was much lower than that of men. However, these results were obtained from crosssectional analyses. The longitudinal data analysis suggested that the rate of physical fitness aging is higher in men $(-0.225)$ than in women $(-0.178)$, which was particularly evident after the age of 80 years old ( -0.367 for men and -0.221 for women). Among men, the rate of change in FAS values was 2.25 times higher those aged $\geq 80$ years than those aged 60-69 years. By contrast, among women, the rate was only 1.2 times higher in those aged $\geq 80$ years than in those aged 60-69 years. From these results, we can deduce that women may possess relatively lower physical fitness than men, but their physical fitness will age at slower rates than in men. The results of this study support the hypothesis reported by Nakamura and Miyao (2008), who stated that "Although women prototypically have less physical strength/health than men at their peak in early adulthood, the rate of decline across their remaining life span is lower than that of their male counterparts". In developed nations, older women often have a longevity advantage over older men (World Health Organization 1989; Health and Welfare Statistics Association 2007), but women suffer from higher levels of morbidity than men (Verbrugge 1982; Hayflick 1994). Therefore, to explain the sex differences in morbidity, healthcare and mortality, intrinsic differences such as genetic factors, sex hormones, and reproductive physiology, and extrinsic differences such as lifestyle, healthy habits, nutrition, and physical fitness should be considered (Nathanson 1977; Verbrugge 1982, 1985). Higher levels of physical fitness may delay the onset of physical disability and loss of independence associated with aging. Thus, we examined the sex differences in physical fitness of older adults to shed some light on the well-documented biological superiority of women in terms of longevity. Clearly, further data are needed to explore these concepts.

The criteria to evaluate fitness markers of aging are somewhat similar with, although slightly fewer in number than, the criteria used to evaluate potential deficit for inclusion in a frailty index (Searle et al. 2008). Major differences between frailty index and FAS is that frailty is a state that becomes more likely the greater the number of deficits that have accumulated (Rockwood and Mitnitski 2007), but physical fitness is just one aspect of it. In the frailty index, pieces of information which might be insignificant on their own sum to give significant results (Kulminski et al. 2008). In contrast, each variables of biomarker of aging should have significant cross-sectional and longitudinal correlations with age and stability. It would be variable to examine the relationship between FAS and frailty, comorbidity, or mortality in further researches. In addition, Rockwood et al. (2007) pointed out the importance of managing missing data if the study includes large population who cannot perform the test. We, however, did not examine the effect of missing data because we used 
the data in healthy elderly who volunteered to participate in the battery test over 7 years.

In conclusion, we identified five fitness markers of aging using step-wise selection method. Using principle component modeling, we predicted individual FAS values from these five fitness markers. The evaluation of physical fitness based on FAS is advantageous and useful because it allows us to estimate the individual's fitness age. Moreover, calculating the slope of the regression line of FAS values onto the CA is indispensable to more precisely determining the rate of physical fitness aging, and was higher in men $(-0.225)$ than in women $(-0.178)$. The results of this study of the longitudinal changes of individual FAS values indicate that older women may have lower physical fitness than older men, but their physical fitness will age more slowly than in men.

Open Access This article is distributed under the terms of the Creative Commons Attribution Noncommercial License which permits any noncommercial use, distribution, and reproduction in any medium, provided the original author(s) and source are credited.

\section{References}

Astrand PO (1956) Human physical fitness with special reference to sex and age. Physiol Rev 36:307-335

Binder EF, Brown M, Sinacore DR, Steger-May K, Yarasheski KE, Schechtman KB (2004) Effects of extended outpatient rehabilitation after hip fracture: a randomized controlled trial. JAMA 292:837-846

Cooper R, Kuh D, Hardy R (2010) Objectively measured physical capability levels and mortality: systematic review and meta-analysis. BMJ 341:c4467

Dubina TL, Mints A, Zhuk EV (1984) Biological age and its estimation. III. Introduction of a correction to the multiple regression model of biological age in cross-sectional and longitudinal studies. Exp Gerontol 19:133-143

Fried LP, Tangen CM, Walston J, Newman AB, Hirsch C, Gottdiener J, Seeman T, Tracy R, Kop WJ, Burke G, McBurnie MA (2001) Frailty in older adults: evidence for a phenotype. J Gerontol A Biol Sci Med Sci 56:M146-M156

Fujita Y, Nakamura Y, Hiraoka J, Kobayashi K, Sakata K, Nagai M, Yanagawa H (1995) Physical-strength tests and mortality among visitors to health-promotion centers in Japan. J Clin Epidemiol 48:1349-1359

Fujita H, Shiomi T, Arahata K, Ishibashi H (2006) Relationship between activity of daily living and motor function in the community-dwelling elderly after hip fracture. Nippon Ronen Igakkai Zasshi 43:241-245 (in Japanese)
Furukawa T, Inoue M, Kajiya F, Inada H, Takasugi S (1975) Assessment of biological age by multiple regression analysis. J Gerontol 30:422-434

Guttman L (1954) Some necessary conditions for common factor analysis. Psychometrika 19:149-161

Harman HH (1967) Modern factor analysis. Chicago University Press, Chicago, pp 68-187

Hayflick L (1994) How and why we age. Ballantine, New York, pp 89-108

Health and Welfare Statistics Association (2007) Physical activity and exercise. Japanese Health and Welfare Statistics 54:82-83 (in Japanese)

Heikkinen E, Kayhty B (1977) Gerontological aspects of physical activity - motivation of older people in physical training. In: Harris R, Frankel LJ (eds) Guide to fitness after fifty. Plenum, New York, pp 191-205

Hofecker G, Skalicky M, Kment A, Niedermuller H (1980) Models of the biological age of the rat. I. A factor model of age parameters. Mech Ageing Dev 14:345-359

Hollingsworth JW, Hashizume A, Jablon S (1965) Correlations between tests of aging in Hiroshima subjects - an attempt to define "physiologic age". Yale J Biol Med 38:11-26

Ingram DK, Nakamura E, Smucny D, Roth GS, Lane MA (2001) Strategy for identifying biomarkers of aging in long-lived species. Exp Gerontol 36:1025-1034

Kaneko M, Morimoto Y, Kimura M, Fuchimoto K (1991) A kinematic analysis of walking and physical fitness testing in elderly women. Can J Sport Sci 16:223-228

Kimura M, Adachi T (1999) Characteristics of fitness and exercise habit in the elderly. J Kyoto Pref Univ Med 9:1-11

Kimura M, Hirakawa K, Okuno T, Oda Y, Morimoto T, Kitani T, Fujita D, Nagata H (1989) An analysis of physical fitness in the aged people with fitness battery test. Jpn J Phys Fitness Sports Med 38:175-185

Kimura M, Hirakawa K, Morimoto T (1990) Physical performance survey in 900 aged individuals. In: Kaneko M (ed) Fitness for the aged, disabled, and industrial worker. Human Kinetics, Champaign, pp 55-60

Kimura M, Arai T, Okayama Y (1994) Ten-year longitudinal evaluation of physical fitness in the elderly. In: Yabe K, Kusano K, Nakata H (eds) Adapted physical activity; health and fitness. Springer, Tokyo, pp 238-242

Kimura M, Okayama Y, Tanaka Y, Kaneko M (1998) Reliability and validity of a simple endurance test for the elderly; shuttle stamina walk test (SSTw). Jpn J Phys Fitness Sports Med 47:401-410

Kulminski AM, Ukraintseva SV, Culminskaya IV, Arbeev KG, Land KC, Akushevich L, Yashin AI (2008) Cumulative deficits and physiological indices as predictors of mortality and long life. J Gerontol A Biol Sci Med Sci 63:1053-1059

Lauretani F, Russo CR, Bandinelli S, Bartali B, Cavazzini C, Di Iorio A, Corsi AM, Rantanen T, Guralnik JM, Ferrucci L (2003) Age-associated changes in skeletal muscles and their effect on mobility: an operational diagnosis of sarcopenia. J Appl Physiol 95:1851-1860

Nakamura E (1994) Statistical approach for the assessment of biological age. In: Balin AK (ed) Practical handbook of human biologic age determination. CRC, Boca Raton, pp 439-456

Nakamura E, Miyao K (2008) Sex differences in human biological aging. J Gerontol A Biol Sci Med Sci 63:936-944 
Nakamura E, Moritani T, Kanetaka A (1989) Biological age versus physical fitness age. Eur J Appl Physiol Occup Physiol 58:778-785

Nakamura E, Moritani T, Kanetaka A (1990) Biological age versus physical fitness age in women. Eur J Appl Physiol Occup Physiol 61:202-208

Nakamura E, Lane MA, Roth GS, Cutler RG, Ingram DK (1994) Evaluating measures of hematology and blood chemistry in male rhesus monkeys as biomarkers of aging. Exp Gerontol 29:151-177

Nakamura E, Moritani T, Kanetaka A (1996) Effects of habitual physical exercise on physiological age in men aged 2085 years as estimated using principal component analysis. Eur J Appl Physiol Occup Physiol 73:410-418

Nakamura E, Lane MA, Roth GS, Ingram DK (1998) A strategy for identifying biomarkers of aging: further evaluation of hematology and blood chemistry data from a calorie restriction study in rhesus monkeys. Exp Gerontol 33:421-443

Nathanson CA (1977) Sex, illness, and medical care. A review of data, theory, and method. Soc Sci Med 11:13-25

Newman AB, Kupelian V, Visser M, Simonsick EM, Goodpaster BH, Kritchevsky SB, Tylavsky FA, Rubin SM, Harris TB, on Behalf of the Health AaBCSI (2006) Strength, but not muscle mass, is associated with mortality in the health, aging and body composition study cohort. J Gerontol A Biol Sci Med Sci 61:72-77

Park MS, Chung SY, Chang Y, Kim K (2009) Physical activity and physical fitness as predictors of all-cause mortality in Korean men. J Korean Med Sci 24:13-19

Piscopo J (1985) Fitness and the aging process: implication for the prevention of illness. Fitness and aging. Wiley, NewYork, pp 96-151

Rockwood K, Mitnitski A (2007) Frailty in relation to the accumulation of deficits. J Gerontol A Biol Sci Med Sci 62:722-727
Rockwood K, Jones D, Wang Y, Carver D, Mitnitski A (2007) Failure to complete performance-based measures is associated with poor health status and an increased risk of death. Age Ageing 36:225-228

Searle SD, Mitnitski A, Gahbauer EA, Gill TM, Rockwood K (2008) A standard procedure for creating a frailty index. BMC Geriatr 8:24

Studenski S, Perera S, Patel K, Rosano C, Faulkner K, Inzitari M, Brach J, Chandler J, Cawthon P, Connor EB, Nevitt M, Visser M, Kritchevsky S, Badinelli S, Harris T, Newman AB, Cauley J, Ferrucci L, Guralnik J (2011) Gait speed and survival in older adults. JAMA, J Am Med Assoc 305:50-58

Suominen H (1978) Effects of physical training in middle-aged and elderly people. In: Komi PV (ed) Studies in sport, physical education and health. Jyvaskyla University Library, Jyvaskyla, pp 1-37

Takata Y, Ansai T, Akifusa S, Soh I, Yoshitake Y, Kimura Y, Sonoki K, Fujisawa K, Awano S, Kagiyama S, Hamasaki T, Nakamichi I, Yoshida A, Takehara T (2007) Physical fitness and 4-year mortality in an 80-year-old population. $\mathrm{J}$ Gerontol A Biol Sci Med Sci 62:851-858

Verbrugge LM (1982) Sex differentials in health. Public Health Rep 97:417-437

Verbrugge LM (1985) Gender and health: an update on hypotheses and evidence. J Health Soc Behav 26:156-182

Voitenko VP, Tokar AV (1983) The assessment of biological age and sex differences of human aging. Exp Aging Res 9:239-244

World Health Organization (1989) Health of the elderly. Report of a WHO Expert Committee, Technical Report Series No779, Geneva, pp. 1-98

Yamada Y, Schoeller DA, Nakamura E, Morimoto T, Kimura M, Oda S (2010) Extracellular water may mask actual muscle atrophy during aging. J Gerontol A Biol Sci Med Sci 65A:510-516 